\title{
COMPETENCIAS PARA UNA INCLUSIÓN DIGITAL EDUCATIVA
}

\section{COMPETENCIES FOR DIGITAL INCLUSION IN EDUCATION}

\section{JORGE BALLADARES-BURGOS ${ }^{1}$}

Recibido: 15 de junio de 2018

Aceptado: 22 de octubre de 2018

\footnotetext{
${ }^{1}$ Pontificia Universidad Católica del Ecuador, Quito, Ecuador, jaballadares@puce.edu.ec
} 
네lㅔ 192 


\section{COMPETENCIAS PARA UNA INCLUSIÓN DIGITAL EDUCATIVA}

\section{COMPETENCIES FOR DIGITAL INCLUSION IN EDUCATION}

Jorge Balladares-Burgos

PALABRAS CLAVE: Inclusión digital, calidad educativa, competencias, TIC

KEYWORDS: Digital inclusion, educational quality, competencies, ICT

\section{RESUMEN}

El presente artículo plantea competencias digitales e informacionales para una inclusión digital educativa tanto dentro como fuera del aula. Se considera que una inclusión digital en la educación brinda pautas y orientaciones para una educación en la diversidad. Una brecha digital desafía a la educación a la bús- queda de igualdad de oportunidades de acceso a la información y al conocimiento para todos. Además, la inclusión digital busca asegurar la calidad educativa, considerando a las TIC como aliadas estratégicas para este fin, y promoviendo un desarrollo de competencias digitales e informacionales en el profesorado. 


\section{ABSTRACT}

The article proposes digital and informational competencies for digital inclusion in learning environments. Digital inclusion in education provides guidance and direction for an education in diversity. A digital divide challenges education to search for equal opportunities for information and knowledge for all. In addition, digital inclusion seeks to ensure educational quality, considering ICT as a strategic tool designed for this purpose, and encouraging the development of e-skills in the academic faculty.

\section{INTRODUCCIÓN}

Actualmente, la educación enfrenta desafíos ante un mundo cambiante. La aparición de las TIC, el internet y las redes sociales inciden en nuestros estilos de vida $y$, por ende, retan a la educación a integrarlas en sus procesos de enseñanza-aprendizaje. La inclusión digital educativa se constituye en uno de esos desafíos para integrar las TIC en los ámbitos educativos con el fin de garantizar una educación de calidad que sea inclusiva y equitativa.

En cuanto a la inserción de las TIC en los procesos educativos en el aula, se percibe que hay una brecha digital entre las generaciones de docentes y las nuevas generaciones estudiantiles (Cobo y Moravec, 2011; Balladares, 2017). Se percibe que las propuestas de capacitación en tecnología educativa para la formación del profesorado no han sido efectivas para generar una integración con la pedagogía y superar su uso instrumental en el aula (Valverde, 2011). Existe una percepción generalizada sobre la calidad de los procesos educativos, dado que lo que se enseña no responde a las necesidades de la vida individual, profesional y en sociedad (Pérez, 2010). La profesión docente se enfrenta, hoy en día, a nuevos desafíos y contextos en una sociedad de la información y en una sociedad nómada del conocimiento (Cobo y Moravec, 2011; Moravec, 2013).

El profesorado se encuentra en la encrucijada de formarse digitalmente para mejorar el proceso educativo ante las nuevas generaciones digitales. Se percibe que los programas de capacitación en el uso de TIC no es suficiente para el desarrollo de competencias digitales en los docentes, y que debería pensarse en una educación digital continua que recupere las buenas prácticas 
cotidianas y una formación permanente con TIC (López, 2005; Valverde-Berrocoso, 2011; Valverde-Berrocoso, Garrido y Fernández, 2010).

El presente artículo tiene como objetivo el plantear competencias para una inclusión digital en la educación. Para este cometido, se plantea la inclusión digital como un concepto y realidad que busca superar la brecha digital ante los crecientes niveles de exclusión y marginalidad producto del avance de la tecnología. Luego, se realiza una aproximación al concepto de inclusión educativa digital, que permita dilucidar una integración efectiva de las TIC en el ámbito de la educación. Finalmente, se proponen competencias digitales e in- formacionales para el profesorado como estrategia de implementar una inclusión digital educativa tanto dentro como fuera del aula.

Se ha realizado una revisión de la literatura de esta temática a partir de investigaciones, artículos científicos y políticas vigentes. La propuesta de este artículo tiene su importancia dado que se considera que la inclusión digital educativa brinda pautas y orientaciones para una educación en la diversidad. Gran parte de esta diversidad que convive la comunidad educativa está mediada por el uso cotidiano de la tecnología y evidencia los diferentes estilos de vida y formas de comprender el entorno humano social y ecológico.

\section{LA INCLUSIÓN DIGITAL COMO SUPERACIÓN DE UNA BRECHA DIGITAL}

Hay diferentes perspectivas sobre el considerar a la inclusión digital como superación de una exclusión digital. El punto de partida se encuentra en la realidad de una brecha digital, que se la puede considerar como un desfase generacional en el acceso a las nuevas tecnologías. Chacón et al. (2017) mencionan que quienes conforman la brecha digital son los ciudadanos que necesitan de la innovación y la tecnología como parte de su vida, pero que, producto de las desigualdades sociales y económicas, no tienen los suficientes medios para acceder a ellos. Esta exclusión digital también se la entiende como la disparidad de los que tienen acceso a internet y de aquellos que no tienen, de quienes poseen un computador frente a quienes no tienen uno. En otras palabras, la brecha digital es la misma brecha social en el mundo digital (Bustamante, 2007). Se intenta eliminar las desigualdades sociales con el desarrollo de las tecnologías de la infor- 
mación y comunicación, pero parecería que la brecha aumenta a medida que se desarrollan nuevas tecnologías y aplicaciones (Bisset, Grossi, \& Borsetti, 2015).

En el caso de Ecuador, estudios relacionados con la reducción de la brecha digital desde el año 2000 al 2015 sitúan al país por debajo de la media internacional en cuanto a conectividad de Internet (Moreira et al., 2017). El Informe sobre la Medición de la Sociedad de la Información (2017) reconoce que todavía existen brechas digitales entre países desarrollados y no desarrollados, a pesar del aumento de la telefonía móvil. Reconoce que también hay una brecha digital entre hombres y mujeres, y entre jóvenes y personas de edad más avanzada.

La inclusión digital aparece como un concepto que pretende la eliminación de la brecha digital. Las transformaciones sociales originadas por las TIC, el internet y las redes sociales han modificado nuestros estilos de vida, de organización y de convivencia, lo que hace necesario garantizar una calidad de vida bajo el principio de dignidad humana. Se puede focalizar a la inclusión digital como un derecho de tercera generación que proviene de los cambios tecnológicos, del avance de la ciencia y su incidencia en la vida humana (Chacón et al., 2017) que otros lo consideran derechos de cuarta generación al momento de la aparición del código abierto para el de- sarrollo educativo y comunitario (UNESCO, 2009). La inclusión digital como derecho humano busca el fomento de la justicia social y, para ello, requiere la colaboración democrática de todos, la generación de procesos innovadores, un diálogo intercultural constructivo y la participación internacional (Lopez \& Samek, 2009).

El derecho a la inclusión digital es un derecho en construcción clave para la democracia hoy en día. Por sí mismo incluye los derechos sociales y los derechos de libertad. A su vez, la inclusión digital cobra vital importancia cuando se busca una universalización del acceso al internet, a la protección de datos y a un gobierno abierto (open government) en función de la transparencia y rendición de cuentas. De hecho, la inclusión digital como derecho no solamente implica reconocer el avance tecnológico innovador que transforma las sociedades, sino también hacer algo por las brechas digital y social que este avance provoca (Torres, 2015). Para otros autores, la inclusión digital se la define como el conjunto de políticas que reconocen la importancia de la inserción de la tecnología en la sociedad. Ella orienta los planes y acciones del Estado para garantizar la conectividad de sus ciudadanos y el acceso a las TIC (Ribeiro, 2013).

Pero las políticas públicas también garantizan la inclusión digital en el ámbi- 
to de la educación. A partir de estos antecedentes de una inclusión digital como derecho humano para superar la brecha digital y las nuevas condiciones de margi- nalidad y exclusión por la falta de acceso a la información y al conocimiento, este artículo aborda una aproximación conceptual a la inclusión digital educativa.

\section{UNA APROXIMACIÓN AL CONCEPTO DE INCLUSIÓN DIGITAL EDUCATIVA}

Desde su etimología la palabra "inclusión" proviene del latín inclusionis y tiene referencia al verbo "incluir", que proviene del latín includere, palabra compuesta por el prefijo in que se traduce como "en" y por claudere con el significado de "cerrar". Por lo tanto, la inclusión implica ese "en-cierro", involucramiento de todos, unión de los diferentes, unidad en la diversidad, universalización de lo particular. A partir de su sentido originario, se puede abordar una comprensión del término inclusión. A su vez, se revisa el tratamiento de los conceptos de inclusión digital e inclusión digital educativa a partir de una aproximación de documentos de organismos internacionales y académicos.

El documento de la UNESCO acerca de una Evaluación de la Educación para Todos (2000) refería a una oferta de educación básica en función de la inclusión y de las necesidades básicas de aprendizaje de niños, adolescentes y jóvenes. En parte, esta inclusión educativa ya se la evidenciaba en el objetivo dos de los
Objetivos de Desarrollo del Milenio (ODM) cuando se planteaba alcanzar la enseñanza primaria universal, y proponía asegurar que los niños y las niñas de todo el mundo puedan terminar un ciclo completo de enseñanza primaria al año 2015.

En cambio, el objetivo cuatro de los Objetivos de Desarrollo Sostenible (ODS) menciona el lograr una educación de calidad, garantizando una educación inclusiva, equitativa y de calidad, con el fin de promover oportunidades de aprendizaje durante toda la vida para todos. Para lograr este cometido, se incluye a todas las personas sin distinción de "sexo, edad, raza, color, pertenencia étnica, idioma, religión, ideas políticas o de otra índole, origen nacional o social, patrimonio o nacimiento, así como las personas con discapacidades, migrantes, pueblos indígenas y niños y jóvenes y, especialmente, quienes se encuentran en situaciones vulnerables o excepcionales" (UNESCO, 2016).

En cuanto a las políticas educativas para la integración de las TIC, es 
importante considerar que algunas de ellas se enfocan a partir de una concepción de equidad, en la que los estudiantes tengan acceso a los recursos con el fin de que logren un máximo nivel de aprendizaje y que desarrollen habilidades y competencias claves para su inserción en una sociedad de la información y el conocimiento. Por ende, este tipo de política debe garantizar la inclusión digital de la población sin distinción de su nivel cultural, geográfico ni social; el soporte educativo a los centros escolares y al profesorado; y una equidad de los resultados de los aprendizajes (Fundación Telefónica, 2018). El Enfoque de la Agenda Educativa Digital (2017) del Ministerio de Educación del Ecuador propone la inclusión digital en la educación promoviendo el acceso a los dispositivos tecnológicos con internet para cada estudiante, así como el desarrollo de competencias pedagógico-digitales para mejorar el aprendizaje de los estudiantes.

A partir de las políticas globales mencionadas, se puede considerar como inclusión educativa al proceso de participación de todos los estudiantes en la comunidad educativa, suprimiendo barreras que limiten el aprendizaje y mejoren la calidad de los centros educativos con la finalidad de que alcancen el éxito escolar (Cabero, 2015). La inclusión educativa es sinónimo de educación en la diversidad, y marca diferencias con visiones puntuales tales como una integración escolar o una ampliación de la educación especial (Cabero \& Córdoba, 2009).

No obstante, hoy en día la incorporación de las Tecnologías de la Información y Comunicación (TIC) en la educación las convierten en aliadas y en estratégicas para alcanzar la calidad y la inclusión educativa cuando el uso de dispositivos móviles permite a los estudiantes acceder a la información y el conocimiento en cualquier tiempo y lugar. Además, los servicios digitales empoderan a docentes y estudiantes a participar en entornos de educación digital. La conectividad proporciona acceso a información para una educación y formación continua de los usuarios (ITU, 2018).

Cabero (2015) menciona tres posibles perspectivas para comprender la inclusión digital en la educación: la primera tiene relación con la utilización de las TIC para favorecer una educación de calidad a través de la eliminación de barreras que impidan a las personas a acceder a la educación, a la cultura y al conocimiento; la segunda busca que los proyectos educativos con TIC se direccionen hacia grupos vulnerables y excluidos con el fin de alcanzar su inclusión educativa, como niños en situación de riesgo y vulnerabilidad, discapacitados, minorías étnicas, entre otros; y la tercera intenta promover una inclusión digital educativa en función de disminuir 
la brecha digital, en el que los niveles bajos de conectividad y de acceso a la información y el conocimiento generan exclusión y analfabetismo digital. Estas nuevas formas de desigualdad y exclusión digital no solamente tienen relación con la conectividad, sino también con la posibilidad de desarrollar competencias digitales e informacionales para la inserción en los nuevos ámbitos laborales diversificados por la mediación de la tecnología.

La inclusión digital educativa posibilita que el uso de las TIC atienda a la diversidad en la educación. Ofrece la alternativa de responder a las exigencias, necesidades, gustos, estilos de vida y demandas de la diversidad de nuestros estudiantes. No se puede desconocer la diversidad de generaciones digitales que surgen mediadas por la aparición de las tecnologías, el internet y las redes sociales (Balladares, 2017). Cabero y Córdoba (2009) mencionan algunas posibilidades que ofrecen las TIC en el ámbito de la diversidad en la educación:

Ayudan a superar las limitaciones que se derivan de las discapacidades cognitivas, sensoriales y motóricas del alumnado. Favorecen la autonomía de los estudiantes, pudiéndose adaptar las necesidades y demandas de cada alumno o alumna de forma personalizada. Ofrecen feed-back in- mediato. Favorecen la comunicación sincrónica y asincrónica de estos estudiantes con el resto de compañeros y el profesorado. Ahorran tiempo en la adquisición de habilidades y capacidades en los estudiantes. Favorecen el diagnóstico de los alumnos y alumnas. Respaldan el modelo de comunicación y de formación multisensorial. Propician una formación individualizada, y el que los alumnos puedan avanzar a su propio ritmo, lo cual es de extrema importancia para los sujetos con algún tipo de discapacidad. Favorecen el desarrollo de la autonomía e independencia de las personas. Evitan la marginación, la brecha digital, que introduce el verse desprovisto de utilizar las herramientas de desarrollo de la sociedad del conocimiento. Facilitan la inserción sociolaboral de aquel alumnado con dificultades específicas. Favorece la disminución del sentido de fracaso académico y personal, los alumnos suelen sentirse más motivados (pp.73-74).

Para Cabero y Córdoba (2009) se requieren de tres condiciones para que se realice una inclusión digital educativa: en primer lugar debe haber una presencia de las TIC; luego, debe haber un acceso y uso de la tecnología; y, por último, se debe generar un proceso 
de alfabetización digital para conocer los lenguajes y sistemas simbólicos de una sociedad de la información y del conocimiento. No obstante, una alfabetización digital es clave para una inclusión digital dado que ella permite que los individuos adquieran y desarrollen habilidades para utilizar las TIC (Kiomi, 2011, citado en Chacón-Penagos et al., 2015). Una educación digital contribuye a una inclusión digital porque permite desarrollar competencias digitales e informacionales para la generación de aprendizajes, gestionar conocimientos y desarrollar competencias generales para el ámbito laboral (Valverde-Berrocoso \& Balladares, 2017).
A partir de esta aproximación conceptual a la inclusión digital educativa, se considera importante buscar estrategias para lograr una integración efectiva de las TIC en los procesos educativos. Nuestros escenarios educativos actuales conviven con estudiantes identificadas con generaciones digitales mediadas por la tecnología móvil, el internet y las redes sociales. Existe un esfuerzo del profesorado por alfabetizarse o educarse digitalmente e incorporar el uso de TIC en sus prácticas. Por este motivo, se realiza una propuesta de competencias digitales e informacionales para una inclusión digital educativa del profesorado, que permita generar una inclusión digital en el aula.

\section{COMPETENCIAS DIGITALES E INFORMACIONALES PARA UNA INCLUSIÓN DIGITAL DEL PROFESORADO}

Las competencias se definen como el conjunto de habilidades, actitudes, aptitudes y valores que una persona refleja en una situación o acontecimiento (Ocampo, Gómez, \& Zambrano, 2015). También se refiere a un saber hacer de manera eficiente, demostrable mediante desempeños observables (Irigoyen, Jiménez, \& Acuña, 2011) o un saber hacer en un contexto (Posada, 2004). Además se desarrollan nuevas acepciones al término como "competencia situada" o "desempeño competente" (Jonnaert, Barrette, Masciotra, \& Yaya, 2006).

Desde la perspectiva del profesorado, Ocampo, Gómez y Zambrano (2015) mencionan que entre las competencias profesionales del docente se encuentran las digitales que hacen referencia al manejo de TIC. Se distinguen varios niveles del desarrollo de la competencia digital, tales como la alfabetización tecnológica, el desarrollo productivo, la integración en ambientes de aprendizaje, 
y las competencias pedagógicas y digitales que permitirán emplear estrategias apoyadas en recursos tecnológicos, como el manejo de herramientas básicas para la docencia, el uso de Internet en la docencia, el diseño de entornos educativos virtuales, el manejo de redes sociales, la evaluación utilizando TIC, entre otros (Ocampo, Gómez, \& Zambrano, 2015).

Se puede entender como competencias digitales a aquellas competencias que permiten una relación con la tecnología para la gestión del conocimiento, la información y la comunicación. No se debe considerar únicamente el desarrollar habilidades digitales a través del uso de TIC, sino que se debe fomentar el uso creativo de las herramientas digitales para la comunicación y el trabajo colaborativo. La competencia digital o e-competencia no se limita a un conjunto de habilidades técnicas como el manejo de hardware y software, sino que también se relacionan con el conocimiento curricular y la pedagogía (Valverde, 2011) Estas competencias digitales se vinculan con el desarrollo del pensamiento creativo, la construcción del conocimiento y el desarrollo de productos innovadores utilizando la tecnología. Este tipo de competencia se la relaciona con el "aprender a aprender" en la cual los recursos tecnológicos son utilizados para desenvolverse en la vida cotidiana, buscar y gestionar la infor- mación de manera autónoma (García \& López, 2012).

Desde la experiencia de la educación infantil y primaria, Quintana (2000) propone algunas competencias que se categorizan como competencias cognitivas, competencias metodológicas y competencias organizativas y de logro. Entre las competencias cognitivas se menciona la capacidad de manejar las TIC e integrarlas a entornos de aprendizaje, la capacidad de utilizar e incorporar las TIC en las actividades de enseñanza-aprendizaje, el conocimiento básico sobre el funcionamiento de equipos informáticos y programas informáticos (en especial, de la web 2.0). En lo concerniente a las competencias metodológicas, se hace referencia a las habilidades para buscar, procesar y analizar información de fuentes digitales, habilidades básicas para el uso del computador, capacidad de evaluar y seleccionar programas de la web 2.0 para fines educativos, y habilidades para el tratamiento y gestión de la información digital. Sobre las competencias organizativas y de logro, se encuentran la capacidad para reflexionar acerca de los usos de los medios tecnológicos en la enseñanza, la habilidad para integrar las TIC en los procesos de enseñanza-aprendizaje, el desarrollo de habilidades para la participación y gestión de proyectos colaborativos a través de redes, y la capacidad de utilizar las 
TIC de manera creativa e innovadora (Quintana, 2000, citado por Del Moral y Villalustre, 2010).

Para Del Moral y Villalustre (2010), la formación del profesor 2.0 debe incluir el desarrollo de competencias tecnológicas para una escuela 2.0 con el fin de ofrecer experiencias didácticas innovadoras y enriquecedoras a los estudiantes. Para lograr una integración curricular de las TIC, optimización de los aprendizajes y mejoramiento de las estrategias de la enseñanza, se proponen las siguientes competencias. Entre ellas, hay que mencionar a las competencias en torno al conocimiento, las relativas al saber. Este tipo de competencias se enfocan en saber dónde, cuándo y cómo utilizar las nuevas tecnologías en el aula; conocer los fundamentos y características de las diferentes herramientas tecnológicas para la formulación de actividades individuales, grupales y colaborativas; adquirir los conocimientos técnicos necesarios sobre recursos y herramientas web para favorecer el aprendizaje.

Por otro lado, se encuentran las competencias en torno a las destrezas, que son relativas al saber hacer. Estas competencias utilizan diferentes aplicaciones y herramientas específicas para cada situación de aprendizaje porque emplean todos los recursos del Internet para fomentar la colaboración entre los estudiantes, el acceso y la gestión de la información a través de la web 2.0. Además, crean y diseñan materiales didácticos y entornos de aprendizaje mediante la utilización de aplicaciones y recursos informáticos que generen nuevos aprendizajes en los estudiantes.

Por último, se distinguen las competencias en torno a las actitudes relativas al saber ser. Ellas propician que los estudiantes apliquen las competencias tecnológicas aprendidas fuera del aula para que ellas se constituyan parte de la vida cotidiana. Además, se actualizan de manera continua, integrando los nuevos recursos, herramientas y metodologías innovadoras en el aula; mostrar inquietud por experimentar, innovar y aprender con las TIC (Del Moral \& Villalustre, 2010).

Desde una perspectiva más pragmática, el portal web Educational Technology and Mobile Learning refiere una serie de competencias digitales para los docentes del siglo XXI. Ese tipo de competencias privilegian el uso y dominio de las TIC, las redes sociales y las diferentes aplicaciones digitales para que sean aplicadas en ámbitos de enseñanza y aprendizaje. 


\section{Tabla 1: Competencias digitales para docentes}

\begin{tabular}{|c|}
\hline Crear y editar audio digital. \\
\hline Utilizar marcadores sociales para compartir los recursos con/entre los estudiantes. \\
\hline Usar blogs y wikis para generar plataformas de aprendizaje en línea dirigidas a sus estudiantes. \\
\hline Usar contenidos audiovisuales y vídeos para involucrar a los estudiantes \\
\hline Usar infografías para estimular visualmente a los estudiantes. \\
\hline Utilizar las redes sociales para conectarse con colegas y crecer profesionalmente. \\
\hline Crear y entregar presentaciones y sesiones de capacitación. \\
\hline Compilar un e-portafolio para su autodesarrollo. \\
\hline Tener un conocimiento sobre seguridad online. \\
\hline Ser capaz de detectar el plagio en los trabajos de sus estudiantes. \\
\hline Crear vídeos con capturas de pantalla y vídeos tutoriales. \\
\hline Recopilar contenido Web apto para el aprendizaje en el aula. \\
\hline Proporcionar a los estudiantes las herramientas de gestión y aprendizaje \\
\hline Conocer el software de votación (por ejemplo, para generar encuestas). \\
\hline Entender las cuestiones relacionadas con derechos de autor y uso honesto de los materiales. \\
\hline Aprovechar los juegos de ordenador y videoconsola con fines pedagógicos \\
\hline Utilizar herramientas digitales para crear cuestionarios de evaluación \\
\hline Uso de herramientas de colaboración para la construcción y edición de textos \\
\hline Encontrar y evaluar el contenido web \\
\hline Usar dispositivos móviles, como tablets o teléfonos inteligentes \\
\hline Identificar recursos didácticos online seguros para los estudiantes \\
\hline Utilizar las herramientas digitales para gestionar el tiempo adecuadamente \\
\hline Conocer el uso de YouTube y sus potencialidades dentro del aula \\
\hline Compartir las páginas web y las fuentes de los recursos que ha expuesto en clase \\
\hline Usar organizadores gráficos, online e imprimibles \\
\hline Aprovechar las herramientas de trabajo online en grupo/en equipo que utilizan mensajería \\
\hline Llevar a cabo un trabajo de investigación utilizando herramientas digitales \\
\hline
\end{tabular}

Fuente: http://www.educatorstechnology.com 
Pozos (2009) considera que la competencia digital debe integrarse al desarrollo profesional del profesor universitario. Además del rol tradicional de docencia, investigación y gestión, el profesor universitario debe incorporar nuevos roles como el de moderador, tutor $u$ orientador de situaciones de aprendizajes presenciales, virtuales o híbridas. Debe considerar también la responsabilidad social con el entorno y el medio ambiente, la seguridad laboral, el manejo de la diversidad, la inclusión, entre otros. A partir de una identificación de un nuevo perfil del docente universitario para una sociedad de la información y el conocimiento, Pozos menciona que se dejará del lado el enfoque meramente instrumental de las tecnologías y se dará paso al docente como profesional que integre las TIC a cada uno de los roles y a su contexto. Para ello, se proponen siete unidades de competencias (Pozos, 2009): planificación y diseño de experiencias de aprendizaje en ambientes presenciales y virtuales; desarrollo y conducción de experiencias de aprendizaje colaborativas presenciales y en red; orientación, guía y evaluación de los procesos de construcción del conocimiento en entornos presenciales y virtuales; gestión del crecimiento y desarrollo profesional con apoyo de las TIC; investigación, desarrollo e innovación pedagógica con/para el uso de las TIC en educación; diversidad, ética y uso responsable de las TIC en el desempeño profesional docente; y medio ambiente, salud y seguridad laboral con el uso de las TIC en la profesión docente.

Carrera y Coiduras (2012) mencionan que el desarrollo de la competencia digital en los estudiantes es posible si el profesorado está capacitado y evidencia un dominio de la competencia para incorporarla en su enseñanza. Entre los componentes principales que configuran la competencia digital del profesorado universitario, se mencionan siete componente: el componente A se relaciona al conocimiento sobre dispositivos, herramientas informáticas y aplicaciones en red, y capacidad para evaluar su potencial didáctico, mientras que el componente $B$ se refiere a al diseño de actividades y situaciones de aprendizaje y evaluación que incorporen las TIC de acuerdo con su potencial didáctico, con los estudiantes y con su contexto. El componente C menciona la implementación y uso ético, legal y responsable de las TIC, mientras que el componente D refleja la transformación y mejora de la práctica profesional docente, tanto individual como colectiva. El componente E habla del tratamiento y la gestión eficiente de la información existente en la red, y el componente F menciona sobre el uso del internet para el trabajo colaborativo y la comunicación e interacción interpersonal. Finalmente, el componente $\mathrm{G}$ 
se refiere a la ayuda proporcionada a los alumnos para que se apropien de las TIC y se muestren competentes en su uso.

En una investigación realizada sobre las competencias digitales más comunes utilizadas por los profesores y estudiantes en el ámbito de las Ciencias Sociales, Carrera y Coiduras (2012) definen 17 elementos de competencias digitales a partir de los componentes principales mencionados

\section{Tabla 2: Diecisiete competencias digitales propuestas}

\begin{tabular}{|l|l|}
\hline \multirow{5}{*}{ Componente A } & Uso de las aplicaciones y funciones del Campus Virtual \\
\cline { 2 - 3 } & Uso de procesadores de texto a nivel de usuario avanzado \\
\cline { 2 - 3 } & Uso de programas de presentaciones a nivel de usuario avanzado \\
\cline { 2 - 2 } & Uso de hojas de cálculo a nivel de usuario avanzado \\
\cline { 2 - 3 } & Uso de herramientas web 2.0 para la edición electrónica en red \\
\hline \multirow{5}{*}{ Componente B } & Acceso a fuentes de documentación en red a partir de buscadores generalistas \\
\cline { 2 - 3 } & Acceso a recursos profesionales y espacios específicos en red relacionados con la formación impartida \\
\cline { 2 - 3 } & Creación de documentos, aplicando el lenguaje multimedia, en soportes y formatos digitales diversos \\
\hline & Aplicación de estrategias de utilización segura de redes \\
\cline { 2 - 3 } & $\begin{array}{l}\text { Actuación respetuosa preservando los derechos de privacidad de las personas en la actividad realizada } \\
\text { en Internet }\end{array}$ \\
\cline { 2 - 3 } & Utilización ética y legal de la información digital \\
\cline { 2 - 3 } & Uso del equipamiento informático aplicando buenas prácticas ergonómicas \\
\hline Componente E & Aplicación de criterios y estrategias de búsqueda y localización eficiente de información en Internet \\
\cline { 2 - 3 } & Uso de agregadores y marcadores sociales para la gestión de información en red \\
\hline Componente F & Uso de las herramientas de comunicación institucional \\
\cline { 2 - 3 } & Presentación eficiente de información multimedia mediante dispositivos específicos \\
\cline { 2 - 3 } & Uso de herramientas para el trabajo colaborativo en red \\
\hline
\end{tabular}

\section{Fuente: Carrera y Coiduras (2012)}

Sobre el desarrollo de competencias digitales se destaca que el profesorado universitario requiere de nuevas alfabetizaciones, en las que se supera un período inicial (primera fase) de alfabetización instrumental en el uso de las tecnologías. Para ello, el profesor universitario debe incorporarse a una segunda 
fase de alfabetización informacional, en las que se incluyan competencias relacionadas con la búsqueda, selección y análisis de información (De Pablos, 2010). Esta alfabetización informacional promueve el saber buscar información en bases de datos o archivos impresos, digitales o audiovisuales, el saber analizar e interpretar información presenta da a través de cualquier formato digital, y el saber producir información y difundirla a través de cualquier formato o tecnología (Marín, Vásquez, Llorente, \& Cabero, 2012).

La finalidad de la competencia informacional consiste en transformar la información en conocimiento. Esta competencia informacional favorece la adquisición de habilidades como buscar la información necesaria, analizar y seleccionar la información de manera eficiente, organizar la información adecuadamente, y utilizar y comunicar la información eficazmente de forma ética y legal. Estas habilidades tendrán como finalidad construir conocimiento (De Pablos, 2010).

El desarrollo de competencias digitales en el profesorado universitario es clave para asegurar una calidad en los procesos educativas en el aula. Por este motivo, luego de una revisión literaria de autores, se proponen las siguientes competencias digitales genéricas para el profesorado universitario:
- Saber diseñar y gestionar actividades de aprendizaje colaborativo presenciales y virtuales.

- Saber desarrollar e innovar estrategias metodológicas con TIC en entornos virtuales de aprendizaje.

- Saber planificar y diseñar actividades de aprendizaje en ambientes presenciales y virtuales.

- Saber acompañar y evaluar procesos de gestión del conocimiento en entornos presenciales y virtuales a través del uso de herramientas digitales y software libre.

- Saber usar de manera responsable las TIC y los entornos virtuales de aprendizaje en el desarrollo profesional docente.

- Saber gestionar el desarrollo profesional docente con apoyo de las TIC y las redes sociales.

- Saber proteger el medio ambiente con el uso de las TIC, información y conocimiento digital.

Como competencias informacionales genéricas para la docencia de la educación superior, se plantean las siguientes:

- Saber seleccionar, analizar e interpretar la información de fuentes de internet.

- Saber generar conocimientos en entornos presenciales y virtuales a 
través del uso de las TIC

- Saber comunicar los resultados de su investigación y práctica docente a través de publicaciones académicas abiertas

(Balladares, 2018).

Estas competencias permiten que el docente desarrolle su capacidad de utilizar las TIC en ámbitos educativos e investigativos. Por ende, la formación y la educación digital del docente serán claves para la incorporación estratégica de las tecnologías de la información y comunicación, no solamente en ámbitos educativos virtuales, sino también en ámbitos educativos presenciales. Así, el profesorado universitario tiene la posibilidad de involucrarse en procesos educativos presenciales, semipresenciales y no-presenciales, e-learning, b-learning o m-learning, fomentar aprendizajes formales, no-formales, ubicuos o invisibles. De esta manera, el desarrollo de competencias digitales e informacionales del profesorado podría garantizar una inclusión digital en el aula en la búsqueda de una calidad educativa.

\section{CONCLUSIONES}

Se puede concluir que las competencias digitales e informacionales contribuyen a una inclusión digital en la educación. Hoy en día, la educación tiene el desafío de responder a la diversidad, y, en este caso, a una diversidad digital evidenciada en las generaciones digitales de niños, adolescentes y jóvenes mediados por la integración de la tecnología en sus diferentes ámbitos de la vida. Aunque los nuevos enfoques educativos mencionen que los procesos educativos se encuentran centrados en el estudiante, no se puede negar el papel fundamental que tiene el docente en la facilitación y mediación de los aprendizajes, en la criticidad y discernimiento ante la abundancia de información, en la gestión del conocimiento y en el ser un referente ético para sus estudiantes. De esta manera se garantiza una calidad en el proceso de enseñanza-aprendizaje mediado por el uso de la tecnología educativa.

La inclusión educativa digital permite realizar una integración efectiva de las TIC en el proceso educativo, en el que el acceso a la información y comunicación mediado por la tecnología y sus diferentes dispositivos, el internet y las redes sociales te abren a un impensado momento de gestión y generación de conocimientos hoy en día. Con ello, los 
aprendizajes no solamente se generan en la formalidad del aula de clase, sino que transcienden el aula y se generan en cualquier espacio y tiempo, por lo que la integración de la tecnología en la vida diaria permite promover esos aprendizajes cotidianos, no-formales e informales.

Los grupos vulnerables, en situación de riesgo y exclusión, y las minorías se vuelven los beneficiarios directos y preferenciales de una inclusión digital educativa. La posibilidad de acceder al conocimiento y la cultura a través del internet y las TIC permite generar oportunidades de acceso a una educación de calidad. Para respaldar lo mencionado, es importante que se considere, a mediano plazo, la inclusión digital como un derecho humano y, a su vez, que la inclusión digital educativa garantice el derecho a la educación de todos.

La reducción de una brecha digital se constituye, actualmente, en un desafío para eliminar aquellas condiciones de exclusión y marginalidad que el avance y el desarrollo de la tecnología producen vertiginosamente. Las políticas nacionales e internacionales deben garantizar a todos los ciudadanos locales y globales el acceso al desarrollo tecnológico que busque el bienestar de los seres humanos en el mundo, una mejor convivencia y comprensión de nuestra diversidad humana y biodiversidad. 


\section{BIBLIOGRAFÎA}

Balladares, J. (2017). Una ética digital para las nuevas generaciones digitales. Revista PUCE(104), 543-563.

Balladares, Jorge (2018). Diseño pedagógico de la educación digital para la formación del profesorado. RELATEC. Revista Latinoamericana de Tecnología Educativa, 17(1), 41-60. DOI: 10.17398/1695288X.17.1.41

Bisset, E., Grossi, A., \& Borsetti, S. (2015). Políticas públicas de inclusión digital: el caso de América Latina y Cuba. Biblios(58). doi:10.5195/biblios.2015.203

Bustamante, J. (2007). Los nuevos derechos humanos: gobierno electrónico e informática comunitaria. Revista Venezolana de Información, Tecnología y Conocimiento, 4(2), 13-27.

Cabero, J. (2015). Inclusión digital-inclusión educativa. Sinergia, 15-18.

Cabero, J., \& Córdoba, M. (marzo de 2009). Inclusión educativa: inclusión digital. Revista Educación Inclusiva, 2(1), 61-77.

Carrera, X., \& Coiduras, J. (2012). Identificación de la competencia digital del profesor universitario: un estudio exploratorio en el ámbito de las Ciencias Sociales. Revista de docencia universitaria, 10(2),
273-298. Obtenido de http:// red-u.net/redu/index.php/REDU/ article/view/383

Centros y Docentes. (2013). Las 33 Competencias Digitales que todo profesor(a) del siglo XXI debiera tener. Obtenido de http:// www.escuela20.com/competencias-skills-habilidades/articulos-y-actualidad/las-33-competencias-digitales-que-todo-profesora-del-siglo-xxi-debiera-tener_2486_42_3979_0_1_in.html

Chacón-Penagos, A. M., Ordóñez-Córdoba, J. A., \& Anichiarico-González, A. M. (enero-junio de 2017). Hacia el reconocimiento de la inclusión digital como un derecho fundamental en Colombia. Vniversi$\operatorname{tas}(134), 139-168$.

De Pablos, J. (2010). Universidad y sociedad del conocimiento. Las competencias informacionales y digitales. Revista de Universidad y Sociedad del Conocimiento, 6-16. Obtenido de http://rusc. uoc.edu/index.php/rusc/article/ view/v7n2-area/v7n2-competencias-informacionales-y-digitales-en-educacion-superior

Del Moral, M., \& Villalustre, L. (2010). Formación del profesor 2.0: desarrollo de 
competencias tecnológicas para la escuela 2.0. Magister. Revista Miscelánea de Investigación, 23, 59-70. Enfoque de la Agenda Educativa Digital (2017). Ministerio de Educación: Quito.

Fundación Telefónica. (2018). Estudio sobre la inclusión de las TIC en los centros educativos de Aulas Fundación Telefónica. Madrid: OEI.

García, M., \& López, R. (2012). Explorando, desde una perspectiva inclusiva, el uso de

las TIC para atender la diversidad. Profesorado. Revista de currículum y formación del profesorado, 277293. Obtenido de http://www. ugr.es/ recfpro/rev161COL4.pdf International Telecommunication Union (2017). Measuring the Information Society

2017. Volume 2. ICT country profiles. Geneva: ITU. Obtenido de https://www.itu.int/en/ITU-D/Statistics/Documents/publications/ misr2017/MISR2017_Volume2. pdf

ITU. (2018). Obtenido de International Telecommunications Union: https://www.itu.int/en/sustainable-world/Pages/goal4.aspx

Moreira, J., Palomares, J., Serrano R. y López, J. (2017). Un breve análisis de la brecha digital de acceso en el Ecuador. Jornadas SARTECO
2017. Zenodo: Ginebra. Obtenido de https://zenodo.org/record/1025815\#.W7T9wddKjce

Ocampo, A., Gómez, M., \& Zambrano, D. (2015). Percepción del profesor sobre el uso del b-learning para fortalecer competencias laborales. Revista Apertura, 7(2). Obtenido de http://www.udgvirtual. udg.mx/apertura/index.php/ apertura/article/view/669

Lopez, P., \& Samek, T. (julio/agosto de 2009). Inclusión digital: un nuevo derecho humano. Educación y Biblioteca(172), 114-118.

Posada, R. (2004). Formación superior basada en competencias, interdisciplinariedad y trabajo autónomo del estudiante. Revista Iberoamericana de Educación. Obtenido de http://rieoei.org/deloslectores/648Posada.PDF

Pozos, K. (27 de junio de 2009). La competencia digital del profesorado universitario para la sociedad del conocimiento: un modelo para la integración de competencia digital en el desarrollo profesional docente. Obtenido de V Congreso de formación para el trabajo: https://www.academia. edu/485126/La_Competencia_ Digital_del_Profesorado_Universitario_para_la_Sociedad_del_ Conocimiento_Un_Modelo_ 
para_la_Integraci\%C3\%B3n_de_ la_Competencia_Digital_en_el_ Desarrollo_Profesional_Docente Regalado, J. (2013). Las competencias digitales en la formación docente. Ra Ximhai,

9(4), 21-29. Obtenido de http://www.revistas.unam.mx/index.php/rxm/ article/download/54013/48091

Ribeiro, F. (2013). Inclusión digital como política pública: disputas en el campo de los Derechos Humanos. Sur. Revista internacional de los Derechos Humanos, 33-55.

Torres, J. (2015). La fundamentación del derecho a la inclusión digital. Revista Prolegómenos. Derechos y Valores, 18(36), 47-64. doi:http:// dx.doi.org/10.18359/dere.933

UNESCO. (1999). Educación para Todos. Evaluación en el Año 2000. Paris: UNESCO.

UNESCO. (22 de Mayo de 2009). UNESCO and Sun Microsystems Announce
Joint Education and Community Development Effort Powered by Open Technologies. UNESCO Press Release(47). Recuperado el 14 de 06 de 2018, de http:// www.lacult.unesco.org/noticias/ showitem.php?uid_ext=calcadmin_1\&getipr=NjYuMjQ5LjY3L$j E 0 \mathrm{Ng}==\& \lg =2 \&$ pais=0\&id=2593

UNESCO. (2016). Desglosar el Objetivo de Desarrollo Sostenible 4. Educación 2030. Guía. Paris: UNESCO. Sector de la Educación.

Valverde, J. (2011). Docentes e-competentes. Barcelona: Octaedro.

Valverde-Berrocoso, J., \& Balladares, J. (2017). Enfoque sociológico del uso del B-learning en la educación digital del docente universitario. Sophia: colección de Filosofía de la Educación, 23(2), 123-140. 